\title{
Native California soils are selective reservoirs for multidrug-resistant bacteria
}

Amanda C. Hollowell, ${ }^{1}$ Kelsey A. Gano, ${ }^{1}$ Gabriel Lopez, ${ }^{1}$ Kareem Shahin, ${ }^{1}$ John U. Regus, ${ }^{1}$ Nathaniel Gleason, ${ }^{1}$ Stefanie Graeter, ${ }^{2}$ Victor Pahua ${ }^{1}$ and Joel L. Sachs ${ }^{1,3 *}$

Departments of ${ }^{1}$ Biology and ${ }^{2}$ Anthropology, and ${ }^{3}$ Institute for Integrative Genome Biology, University of California, Riverside, CA, USA.

\section{Summary}

Soil bacteria can exhibit extensive antibiotic resistomes and act as reservoirs of important antibiotic resistance traits. However, the geographic sources and evolutionary drivers of resistance traits are poorly understood in these natural settings. We investigated the prevalence, spatial structure and evolutionary drivers of multidrug resistance in natural populations of Bradyrhizobium, a cosmopolitan bacterial lineage that thrives in soil and aquatic systems as well as in plant and human hosts. We genotyped $>400$ isolates from plant roots and soils across California and assayed 98 of them for resistance traits against 17 clinically relevant antibiotics. We investigated the geographic and phylogenetic structure of resistance traits, and analysed correlations of resistance with strain abundance, host infection capacity and in vitro fitness. We found: (i) multidrug resistance at all sites, (ii) subsets of resistance traits that are spatially structured and (iii) significant associations between resistance traits and increased strain abundance or host infection capacity. Our results highlight multiple selective factors that can result in the spread of resistance traits in native Bradyrhizobium populations.

\section{Introduction}

Antibiotic resistance is ancient in bacterial populations, and the origins of most resistance traits predate human selective pressures (D'Costa et al., 2011). In natural settings, resistance traits are thought to be important in modulating inter-microbial communication and conflict (Martinez, 2008) irrespective of any anthropogenic effects

Received 10 September, 2014; accepted 16 December, 2014.

*For correspondence. E-mail joels@ ucr.edu; Tel. +1 951827 6357; Fax +19518274286 .

(C) 2015 Society for Applied Microbiology and John Wiley \& Sons Ltd
(Singer et al., 2006). Native bacterial communities can exhibit extensive 'natural resistomes' (D'Costa, 2006; Nesme et al., 2014), but little is known about the abundance, spatial distribution or selective drivers of multidrug resistance traits. Here, we investigated the population genetics and evolution of antibiotic resistance in Bradyrhizobium (alphaproteobacteria). Bradyrhizobium are cosmopolitan bacteria that thrive in soils and aquatic environments (Chaintreuil et al., 2000), and are found associated with plants (Vinuesa et al., 2005; 2008; Sachs et al., 2009) and animals, including humans (Hunt et al., 2011; Bhatt et al., 2013; Costello et al., 2013). Bradyrhizobium vary in antibiotic resistance (Mueller et al., 1988) and can be multidrug resistant (Cole and Elkan, 1979). Multiple putative resistance proteins are annotated within Bradyrhizobium genomes, but without predicted specificity to any antibiotic in most cases (http://genome.microbedb.jp/rhizobase/ Bradyrhizobium). Moreover, Bradyrhizobium genomes contain low proportions of 'core' proteins (i.e. proteins that are universally shared among strains $23-33 \%$ ), so strains can vary a great deal in gene content and are often sparsely annotated (Tian et al., 2012). We genotyped Bradyrhizobium isolates cultured from root nodules and the soil-root interface of native California Lotus species (herbaceous legumes) and assessed resistance to 17 antibiotics. We investigated the phylogenetic, population genetic and geographic structure of resistance traits, as well as the correlation of resistance with strain abundance, nodulation capacity, in vitro fitness and geographic distribution.

\section{Results and discussion}

Isolation of Bradyrhizobium and antibiotic resistance assay

Isolates were cultured from the root nodules and the soil-root interface of Lotus strigosus following published protocols (Sachs et al., 2009) (Appendix S1). We included previously cultured isolates from $L$. strigosus, $L$. micranthus, $L$. heermannii and $L$. wrangelianus (Sachs et al., 2009) (Table S1). Most isolates (301/417) were collected from gated preserves with negligible human impact, 37 isolates were collected from sites in state parks and 79 isolates were collected in a canyon with limited access, but close to housing development (Table S1). We 
chose a representative subset of $98 / 417$ focal isolates to test for antibiotic resistance and other traits. We initially studied isolates from the Bodega Marine Reserve (Sachs et al., 2009), then expanded this study to multiple sites attempting to sample diverse soils and to maximize genetic diversity among collection sites (Table S1).

Seventeen different antibiotics were tested, including ampicillin (Acros Organics, Morris Plains, NJ, USA); carbenicillin, ciprofloxacin, erythromycin, gentamicin, hygromycin B, kanamycin, neomycin, penicillin, rifampicin (Fisher Scientific, Pittsburgh, PA, USA); chloramphenicol, spectinomycin, streptomycin (MP Biomedical, Santa Ana, CA, USA); doxycycline, novobiocin, paromycin, and tetracycline (Sigma-Aldrich, St Louis, MO, USA). Two researchers independently assayed growth of the focal isolates on plates with modified arabinose gluconate (MAG) (Sachs et al., 2009) containing one of 17 antibiotics at four different concentrations $(25,50,100$ and $200 \mu \mathrm{g} \mathrm{ml}^{-1}$ ). Sterile toothpicks were used to create streaks on antibiotic plates, followed by control plates, in randomly assigned locations (Haahr, 1998). Antibiotic and control plates were incubated at $29^{\circ} \mathrm{C}$, photographed and scored on the fifth day following plating. Streaks on antibiotics were compared with the respective control streaks, and binary assignments of resistance were recorded (resistance $=$ growth on the plate; sensitivity $=$ no growth).

Two antibiotics did not exhibit variation in resistance. All isolates were sensitive to novobiocin and were resistant to erythromycin (Table S2). At the conservative cut-off of $100 \mu \mathrm{g} \mathrm{ml}^{-1}$ to assign resistance, we failed to find resistance traits to only four antibiotics (ciprofloxacin, novobiocin, spectinomycin and tetracycline) (Fig. 1). Moreover, we found multidrug-resistant Bradyrhizobium to be ubiquitous in native California soils, even in pristine settings (Table S2). At the conservative $100 \mu \mathrm{g} \mathrm{ml}^{-1}$ cut-off for resistance, we found isolates to be resistant to an average of four antibiotics and uncovered isolates that were resistant to up to 10 antibiotics (Fig. 2). The most common $4 \times$ resistance trait combination (chloramphenicol, erythromycin, neomycin and paromycin) was uncovered in 21 isolates from all collection sites (Table S2). Most isolates (76/82) were resistant to at least two unrelated classes of antibiotics. Thus, patterns of multidrug resistance were not driven by cross-resistance against related antibiotics. Our data support previous research that showed intrinsic antibiotic resistance in Bradyrhizobium, and demonstrates the ubiquity of multidrug resistance in wild Bradyrhizobium populations.

\section{Phylogenetic tree reconstruction}

For each isolate, we amplified five loci. Evolutionary relationships were reconstructed among the focal strains using three chromosomal loci (gInll, ITS, recA) (van Berkum and Fuhrmann, 2000; Vinuesa et al., 2005; Sachs et al., 2011), and separately using two loci on a genomic island that encodes symbiosis traits (nifD and nodD-A) (Sachs et al., 2010), because this 'symbiosis island' can be horizontally transferred among Bradyrhizobium lineages (Sullivan and Ronson, 1998). Phylogenetic trees were reconstructed using RAXML (GUI1.3) (Silvestro and Michalak, 2012) with the following parameters: maximum likelihood, 1000 rapid bootstrap replicates, per-partition branch lengths and a GTRgamma nucleotide substitution model using partitioned datasets. The chromosomal phylogenetic tree recovered multiple Bradyrhizobium species lineages (Fig. 3, Appendix S1). Isolates can be

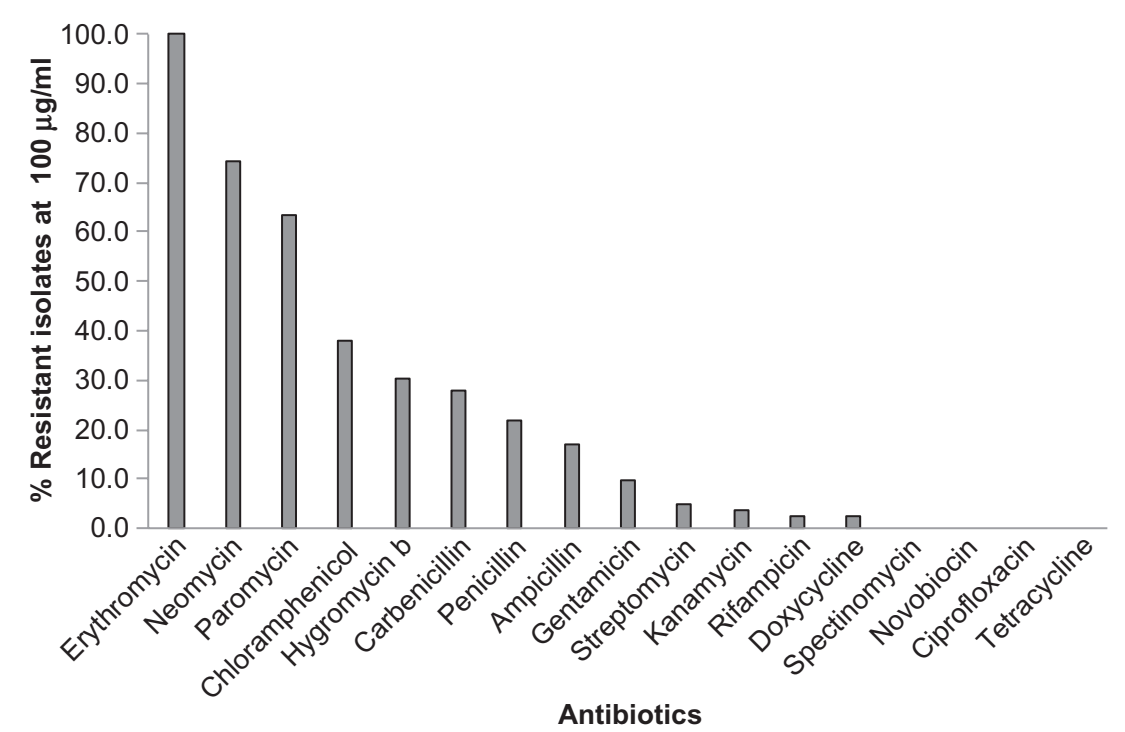

Fig. 1. Population frequency of resistance traits among antibiotics. Resistant Bradyrhizobium isolates were uncovered for all but four antibiotics at $100 \mathrm{ug} \mathrm{ml}^{-1}$ (spectinomycin, novobiocin, ciprofloxacin, tetracycline).

(C) 2015 Society for Applied Microbiology and John Wiley \& Sons Ltd, Environmental Microbiology Reports, 7, 442-449 


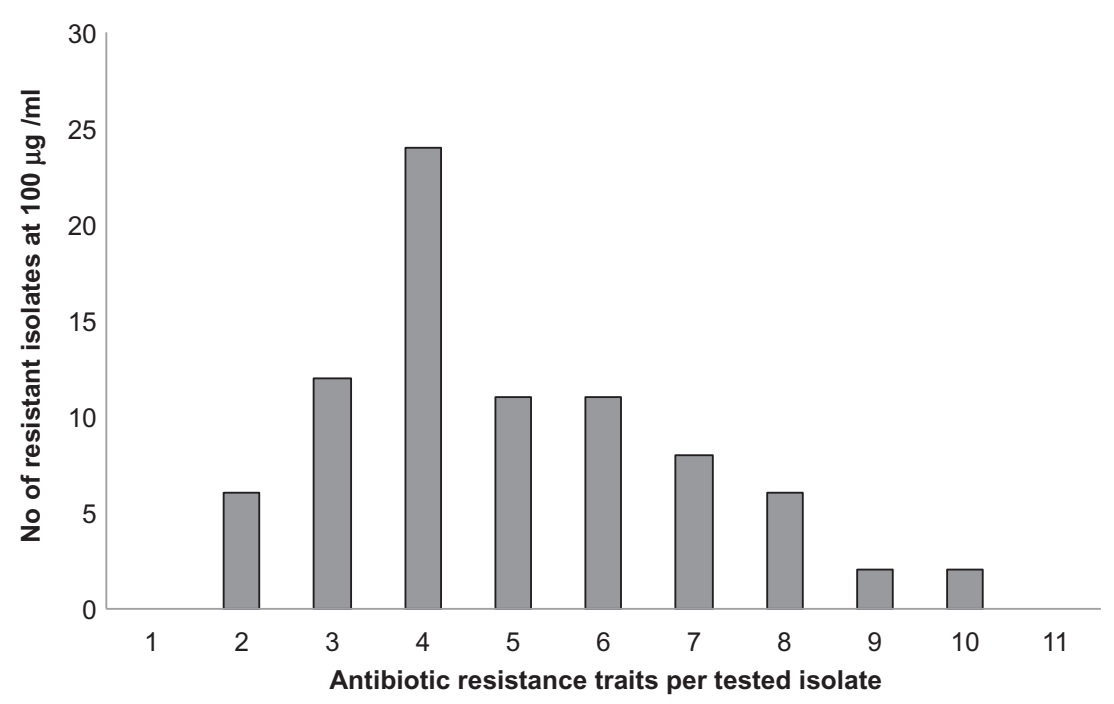

Fig. 2. Population frequency of multidrug antibiotic resistance: Grey bars indicate the number of isolates that are resistant to the given number of antibiotics at $100 \mu \mathrm{g} \mathrm{ml}^{-1}$.

assigned to the following species: B. canariense, $B$. japonicum, B. liaoningense and B. retamae using monophyletic groups with high branch support $(>0.9)$. Some isolates do not form highly supported monophyletic groups with known reference species and could be novel lineages.

\section{Population genetic analyses}

We analysed molecular diversity indices and interpopulation variation (FST) using Arlequin (Excoffier and Lischer, 2010) for concatenated $g / n / l$, ITS and $\operatorname{rec} A$ sequences (2686 bp). Gene diversity (Nei, 1987) was high within all eight populations (all $>0.8333$ ) (Table S3). The Anza Borrego State Park site was greatly differentiated from all other populations, and the other between population FST values were often not significant (17/28; Tables S4 and S5). This is likely due to the small sample sizes at some collection sites.

\section{Data independence tests}

Multilocus sequence typing of the 98 focal isolates uncovered several isolates with identical sequences (haplotypes). To test whether these isolates were independent for resistance traits, we compared three potential sources of variation in antibiotic resistance score (experimenter gathering the data, different isolates with the same haplotype and different isolates with different haplotypes). Isolates with identical haplotypes were non-independent for antibiotic resistance, exhibiting similar variation in resistance scores to identical isolates tested by different experimenters (Pearson's $\chi^{2}=16.109$, $P=0.1371, d f=11$ ), and significantly less variation than isolates with different haplotypes (Pearson's $\chi^{2}=38.842$, $P=0.0019, d f=17$ ) (Table S6). Thus, a single isolate per each haplotype was selected for further analyses using a random number generator (Haahr, 1998), resulting in a total of 82 unique haplotypes.

Resistance to antibiotics of the same class can be non-independent due to structural similarity. To assess independence, we compared two sources of variation (pairs of antibiotics of the same class versus different classes). Variation in resistance scores within antibiotic classes was not significantly different from variation in resistance among antibiotic classes (Pearson's $\chi^{2}=$ 292.389, $P=0.5156, d f=294$ ) (Table S6). Thus, all antibiotics were treated as independent.

\section{Correlated evolution of antibiotic resistance traits with geographic location, abundance, nodulation capacity and colony size}

We tested antibiotic resistance traits, colony growth rates and nodulation capacity for phylogenetic signal by quantifying Pagel's lambda ( $\lambda$; Pagel, 1999) and Fritz and Purvis' D (Fritz and Purvis, 2010) (Table S7). These tests are key prerequisites for the evolutionary analyses that follow. Resistance to hygromycin B, neomycin and chloramphenicol exhibited statistically significant phylogenetic signal for both Pagel's lambda and Fritz Purvis' D. Kanamycin, paromycin and streptomycin resistance exhibited statistically significant phylogenetic signal for Pagel's lambda only and gentamicin exhibited phylogenetic signal for Fritz Purvis' D only. Symbiotic capacity exhibited statistically significant phylogenetic signal for both Pagel's lambda and Fritz Purvis' D. Colony growth rate on MAG and YM media exhibited statistically significant phylogenetic signal for Pagel's lambda (Table S7). Even low levels of horizontal gene transfer can eliminate statistical dependence upon the phylogenetic tree, as occurs in cophylogenetic analyses 


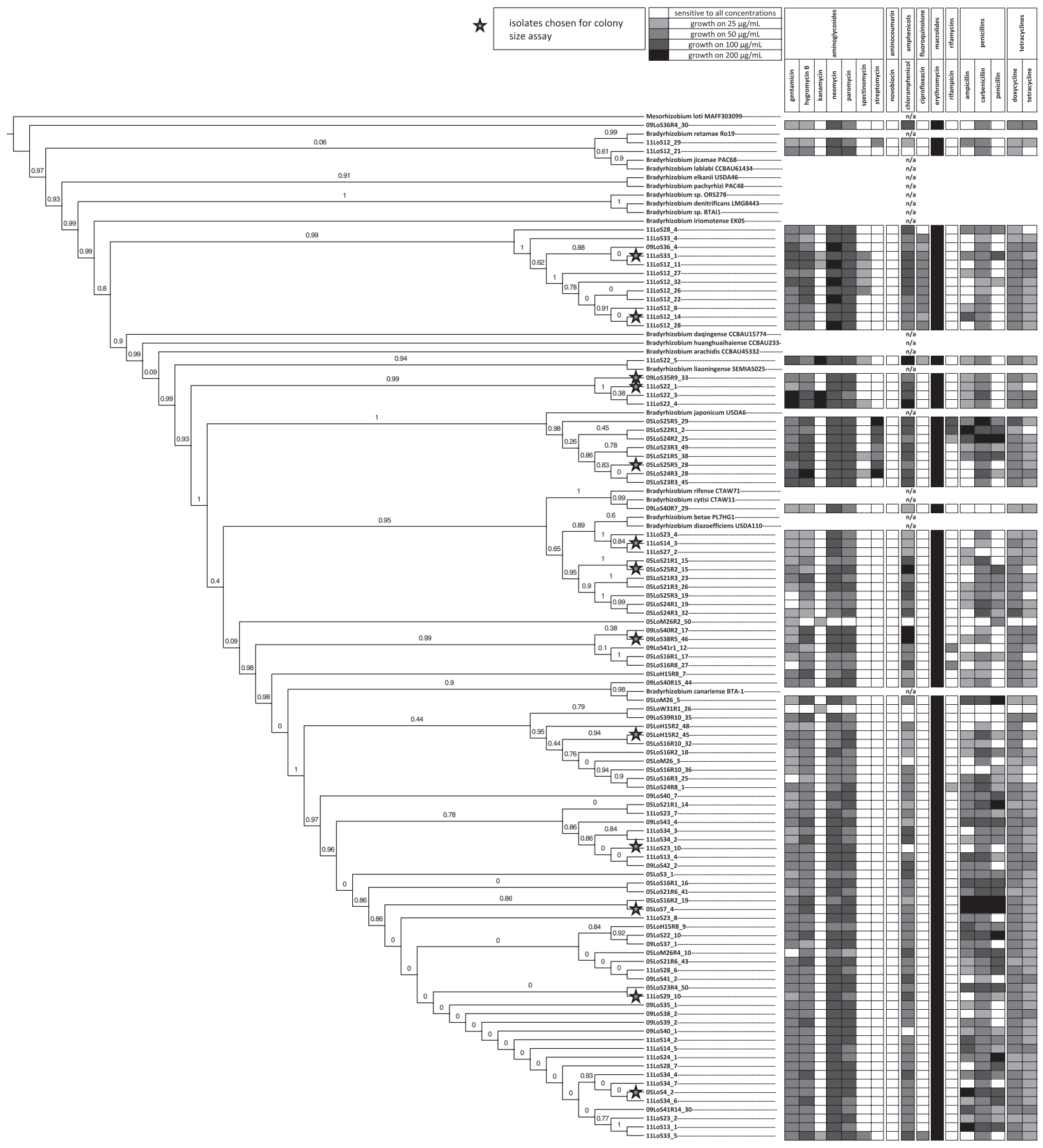

Fig. 3. Maximum likelihood cladogram reconstructed from concatenated glnIl, ITS and recA loci from 82 isolates with corresponding heat map of antibiotic resistance. White indicates sensitivity at all tested concentrations while black indicates resistance at all tested concentrations. Isolates chosen for colony size assessment on multiple solid media types are phylogenetically diverse as indicated by stars.

of horizontally transferred symbionts and their hosts (Schilthuizen and Stouthamer, 1997). Thus, traits with significant phylogenetic signal (resistance to chloramphenicol, gentamicin, hygromycin B, kanamycin, neomy- cin, paromycin, streptomycin; largely aminoglycosides) (Table S7) are most likely faithfully passed from parent to offspring. Traits without significant phylogenetic signal (resistance to ampicillin, carbenicillin, doxycycline, 
penicillin and rifampicin) (Table S7) might be rapidly evolving, swiftly being lost due to local selection, or evolving via horizontal gene transfer.

Geographic sites. Resistance traits might be selectively favoured in soils depending on local soil microbiota or other factors that shape resistance traits (Singer et al., 2006). Associations between quantitative antibiotic resistance (maximum concentration of resistance) among the seven field collection sites were tested using chi-squared tests (likelihood ratio and Pearson's) in JMP (SAS Institute Inc, 1989). Quantitative antibiotic resistance traits exhibited non-random spatial structure among geographic sites for six resistance traits, using both likelihood ratio and Pearson's chi-squared tests (Pearson's; ciprofloxacin, $\chi^{2}=59.274, \quad P<0.0001$, $d f=12$; kanamycin, $\chi^{2}=37.177, \quad P=0.0002, \quad d f=12$; neomycin, $\chi^{2}=61.148, P<0.0001, d f=18$; penicillin, $\chi^{2}=37.762, \quad P=0.0367, \quad d f=24 ; \quad$ spectinomycin, $\chi^{2}=36.449, \quad P=0.0003, \quad d f=12 ; \quad$ tetracycline,$\quad \chi^{2}=$ 30.479, $P=0.0024, d f=12$ ). Resistance to carbenicillin was statistically significant for the likelihood ratio chi-squared test only $\left(\chi^{2}=40.556, P=0.0186, d f=24\right)$ (Table S8). Among resistance traits with spatial structure, ciprofloxacin, kanamycin, neomycin and spectinomycin resistance were mostly localized at inland desert populations (Anza Borrego Desert State Park, Burns Piñon Ridge Reserve) but also at Bodega Marine Reserve and San Dimas Canyon (Fig. S1A-D). Resistance to higher concentrations of tetracycline was localized to inland Southern California (Fig. S1E) and a pattern of higher resistance in the western populations was observed for carbenicillin (Fig. S1F). Significant geographical structure of resistance traits is consistent with localized or regional selective pressures favouring the spread of resistance traits (e.g. carbenicillin, ciprofloxacin, kanamycin, neomycin, penicillin, spectinomycin and tetracycline) (Table S8, Fig. S1A-G). Although each sampled population was genetically diverse (Table S3), some collection sites have small samples and it is possible that these patterns are driven by relatively few isolates. However, the high gene diversity found across populations $(>0.8333$ ) indicates that our sampling captured variation in the populations.

Plant root nodulation capacity. Resistance traits might be advantageous for bacterial genotypes that compete in the rhizosphere to infect plant hosts. Root-nodule forming genotypes of Bradyrhizobium can form intra-cellular infections on legumes, whereas many Bradyrhizobium genotypes do not have this capacity (and persist in the soil or the soil-root interface). Previous work found mixed results for a relationship between antibiotic resistance and nodulation capacity (Schwinghamer, 1964; Levin and
Montgomery, 1974; Turco et al., 1986), but these studies used mutant strains. Here, nodulation capacity was assessed using two different methods. Some isolates were previously inoculated on L. strigosus [a permissive host with a broad symbiont range (Sachs et al., 2009)] to confirm nodulation capacity. For the remainder, nodulation capacity was assessed via polymerase chain reaction (PCR) amplification of the symbiosis loci nifD and nodD-A. Amplification of both of these loci is consistent with a root-nodulating strain, whereas soil isolates that lack amplifiable DNA in both these symbiosis-island loci consistently fail to form nodules on $L$. strigosus (Sachs et al., 2010; 2011) (Table S2). For antibiotic resistance traits exhibiting no phylogenetic signal, associations between nodulation capacity and antibiotic resistance traits (100 $\mu \mathrm{g} \mathrm{ml}^{-1}$ cut-off) were tested with chi-squared tests (likelihood ratio and Pearson's) in JMP (SAS Institute Inc, 1989). For antibiotics exhibiting phylogenetic signal, we used the likelihood ratio test with the ML option of the discrete function in BayesTraits (Pagel and Meade, 2006). We uncovered support for the dependent evolution of hygromycin $B$ and neomycin resistance with rootnodulation capacity (hygromycin, $D=13.26533, P=0.01$, $d f=4$; neomycin, $D=20.317124, \quad P=0.0004, \quad d f=4$ ) (Table S9). Additionally, evolutionary transitions rates among these states (nodulation capacity, hygromycin B resistance) support a correlation between nodulation capacity and antibiotic resistance (Fig. S2A and B).

Haplotype abundance. Resistance traits might also be advantageous for competition and spread in the environment. Soil bacteria often exhibit a highly skewed distribution with many rare haplotypes and a handful of highly abundant ones (McInnes, 2004). We issued binary abundance assignments (haplotypes with $\geq$ five isolates and constituting $>1 \%$ of the sampled population were considered abundant and the remainder of haplotypes were considered 'rare'). For antibiotic resistance traits exhibiting no phylogenetic signal, associations between abundance and antibiotic resistance traits $\left(100 \mu \mathrm{g} \mathrm{ml}^{-1}\right.$ cut-off) were tested with chi-squared tests (likelihood ratio and Pearson's) in JMP (SAS Institute Inc, 1989). For antibiotics exhibiting phylogenetic signal, we used the likelihood ratio test with the $\mathrm{ML}$ option of the discrete function in BayesTraits (Pagel and Meade, 2006). Ampicillin resistance was associated with increased abundance (ampicillin, $\chi^{2}=6.005, P$-value $\left.=0.0143, d f=1\right)($ Table S10 $)$ and likelihood ratio tests significantly support the dependent evolution of abundance and neomycin resistance traits $(D=10.88488, P=0.0279, d f=4)$. Similarly, most transitions rates among these states (high versus low abundance, neomycin resistance) support a correlation between increased abundance and neomycin resistance (Fig. S2C). 
Colony growth rate. Antibiotic resistance can often bear costs for bacterial growth (Andersson and Levin, 1999). We examined correlations between resistance scores at the $100 \mathrm{\mu g} \mathrm{ml}^{-1}$ cut-off and colony growth rate as measured by average colony size on each of three different media [GRDM (Sullivan et al., 1996), YM (Somasegaran et al., 1994) and MAG (Sachs et al., 2009)] in a phylogenetically diverse subset of 13 strains (Fig. 3). We used either analysis of variance in JMP (SAS Institute Inc, 1989) or phylogenetic independent contrasts using the crunch command in the Caper package (Orme, 2012) of $R$ (R Core Team, 2013) based on if the antibiotic resistance trait exhibited phylogenetic signal. Colony growth rates on three media types uncovered no consistent evidence for costs to antibiotic resistance. On GRDM, resistance to hygromycin $B$ was correlated with smaller colony size $(F=54.120, P=0.000)$ while paromycin resistance was correlated with larger colony size $(F=34.700, P=0.000)$. The results were converse on MAG (hygromycin $B$ : $\mathrm{F}=16.570, P=0.001$; paromycin: $\mathrm{F}=52.420, P=0.000$ ) and $\mathrm{YM}$ (hygromycin $\mathrm{B}: \mathrm{F}=17.880, \quad P=0.008$; paromycin: $F=7.474, P=0.017)$. A further two antibiotics exhibited statistically significant correlations between antibiotic resistance and colony size on YM only (gentamicin: $\mathrm{F}=9.669, P=0.008$; streptomycin: $\mathrm{F}=6.969, P=0.020$ ) (Table S11). Although some resistance traits exhibited significant associations with colony growth rate (Table S11), no overall pattern was apparent.

\section{Conclusion}

In native Bradyrhizobium populations, we uncovered resistance traits for nearly all antibiotics tested, and also found multidrug resistance to be ubiquitous. Antibiotic resistance traits differed in their phylogenetic signal and likely have differing evolutionary trajectories with some being passed faithfully from parent to offspring and others capable of horizontal gene transfer. Antibiotic resistance traits also differed in their evolutionary drivers. Some antibiotics exhibited spatial structure suggesting local selection in soils, while others had correlated evolution with Bradyrhizobium haplotype abundance or plant root nodulation capability indicating that multiple factors drive selection for and maintenance of these resistance traits.

\section{Acknowledgements}

The following grants supported this study: to $\mathrm{ACH}$ a Herbert Kraft Scholarship and a UC Riverside Graduate Research Mentorship Fellowship, to GL a UC Riverside Science Circle Student Award, to KS a UC Riverside CNAS Dean's Research Fellowship, to JLS NSF DEB 0816663 and NSF DEB 1026424

\section{References}

Andersson, D.I., and Levin, B.R. (1999) The biological cost of antibiotic resistance. Curr Opin Microbiol 2: 489493.

Bhatt, A.S., Freeman, S.S., Herrera, A.F., Pedamallu, C.S., Gevers, D., Duke, F., et al. (2013) Sequence-based discovery of Bradyrhizobium enterica in cord colitis syndrome. New Engl J Med 369: 517-528.

Chaintreuil, C., Giraud, E., Prin, Y., Lorquin, J., Ba, A., Gillis, M., et al. (2000) Photosynthetic bradyrhizobia are natural endophytes of the African wild rice Oryza breviligulata. Appl Environ Microbiol 66: 5437-5447.

Cole, M.A., and Elkan, G.H. (1979) Multiple antibiotic resistance in Rhizobium japonicum. Appl Environ Microbiol 37: 867-870.

Costello, E.K., Carlisle, E.M., Bik, E.M., Morowitz, M.J., and Relman, D.A. (2013) Microbiome assembly across multiple body sites in low-birthweight infants. mBio 4: e00782-13e00782-13.

D'Costa, V.M. (2006) Sampling the antibiotic resistome. Science 311: 374-377.

D'Costa, V.M., King, C.E., Kalan, L., Morar, M., Sung, W.W.L., Schwarz, C., et al. (2011) Antibiotic resistance is ancient. Nature 477: 457-461.

Excoffier, L., and Lischer, H.E.L. (2010) Arlequin suite ver 3.5: a new series of programs to perform population genetics analyses under Linux and Windows. Mol Ecol Resour 10: $564-567$.

Fritz, S.A., and Purvis, A. (2010) Selectivity in mammalian extinction risk and threat types: a new measure of phylogenetic signal strength in binary traits. Conserv Biol 24: 1042-1051.

Haahr, M. (1998) RANDOM.ORG - True Random Number Service. [WWW document]. URL http://www.random.org.

Hunt, K.M., Foster, J.A., Forney, L.J., Schütte, U.M.E., Beck, D.L., Abdo, Z., et al. (2011) Characterization of the diversity and temporal stability of bacterial communities in human milk. PLOS ONE 6: e21313.

Levin, R.A., and Montgomery, M.P. (1974) Symbiotic effectiveness of antibiotic-resistant mutants of Rhizobium japonicum. Plant Soil 41: 669-676.

Mclnnes, A. (2004) Structure and diversity among rhizobial strains, populations and communities? A review. Soil Biol Biochem 36: 1295-1308.

Martinez, J.L. (2008) Antibiotics and antibiotic resistance genes in natural environments. Science 321: 365367.

Mueller, J.G., Skipper, H.D., Shipe, E.R., Grimes, L.W., and Wagner, S.C. (1988) Intrinsic antibiotic resistance in Bradyrhizobium japonicum. Soil Biol Biochem 20: 879882.

Nei, M. (1987) Molecular Evolutionary Genetics. New York, NY, USA: Columbia University Press.

Nesme, J., Cécillon, S., Delmont, T.O., Monier, J.-M., Vogel, T.M., and Simonet, P. (2014) Large-scale metagenomicbased study of antibiotic resistance in the environment. Curr Biol 24: 1096-1100.

Orme, D. (2012) The caper package: Comparative analysis of phylogenetics and evolution in $\mathrm{R}$.

Pagel, M. (1999) Inferring the historical patterns of biological evolution. Nature 401: 877-884. 
Pagel, M., and Meade, A. (2006) Bayesian analysis of correlated evolution of discrete characters by reversible-jump Markov chain Monte Carlo. Am Nat 167: 808-825.

R Core Team (2013) R: A language and environment for statistical computing R Foundation for Statistical Computing, Vienna, Austria.

Sachs, J.L., Kembel, S.W., Lau, A.H., and Simms, E.L. (2009) In Situ phylogenetic structure and diversity of wild Bradyrhizobium communities. Appl Environ Microbiol 75: 4727-4735.

Sachs, J.L., Ehinger, M.O., and Simms, E.L. (2010) Origins of cheating and loss of symbiosis in wild Bradyrhizobium. $J$ Evol Biol 23: 1075-1089.

Sachs, J.L., Russell, J.E., and Hollowell, A.C. (2011) Evolutionary instability of symbiotic function in Bradyrhizobium japonicum. PLoS ONE 6: e26370.

SAS Institute Inc (1989) JMP SAS Institute Inc., Cary, NC, USA.

Schilthuizen, M.O., and Stouthamer, R. (1997) Horizontal transmission of parthenogenesis-inducing microbes in Trichogramma wasps. Proc R Soc B 264: 361-366.

Schwinghamer, E.A. (1964) Association between antibiotic resistance and effectiveness in mutant strains of Rhizobium spp. Can J Microbiol 10: 221-233.

Silvestro, D., and Michalak, I. (2012) raxmIGUI: a graphical front-end for RAxML. Org Divers Evol 12: 335-337.

Singer, R.S., Ward, M.P., and Maldonado, G. (2006) Can landscape ecology untangle the complexity of antibiotic resistance? Nat Rev Micro 4: 943-952.

Somasegaran, P., Hoben, H.J., and Somasegaran, P. (1994) Handbook for Rhizobia. New York, NY: Springer-Verlag.

Sullivan, J.T., and Ronson, C.W. (1998) Evolution of rhizobia by acquisition of a 500-kb symbiosis island that integrates into a phe-tRNA gene. PNAS 95: 5145-5149.

Sullivan, J.T., Eardly, B.D., van Berkum, P., and Ronson, C.W. (1996) Four unnamed species of nonsymbiotic rhizobia isolated from the rhizosphere of Lotus corniculatus. Appl Environ Microbiol 62: 2818-2825.

Tian, C.F., Zhou, Y.J., Zhang, Y.M., Li, Q.Q., Zhang, Y.Z., Li, D.F., et al. (2012) Comparative genomics of rhizobia nodulating soybean suggests extensive recruitment of lineage-specific genes in adaptations. PNAS 109: 86298634.

Turco, R.F., Moorman, T.B., and Bezdicek, D.F. (1986) Effectiveness and competitiveness of spontaneous antibiotic-resistant mutants of Rhizobium leguminosarum and Rhizobium japonicum. Soil Biol Biochem 18: 259262.

van Berkum, P., and Fuhrmann, J.J. (2000) Evolutionary relationships among the soybean bradyrhizobia reconstructed from 16S rRNA gene and internally transcribed spacer region sequence divergence. Int J Syst Evol Microbiol 50: 2165-2172.

Vinuesa, P., Silva, C., Werner, D., and Martínez-Romero, E. (2005) Population genetics and phylogenetic inference in bacterial molecular systematics: the roles of migration and recombination in Bradyrhizobium species cohesion and delineation. Mol Phylogenet Evol 34: 29-54.

Vinuesa, P., Rojas-Jimenez, K., Contreras-Moreira, B., Mahna, S.K., Prasad, B.N., Moe, H., et al. (2008)
Multilocus sequence analysis for assessment of the biogeography and evolutionary genetics of four Bradyrhizobium species that nodulate soybeans on the Asiatic continent. Appl Environ Microbiol 74: 6987-6996.

\section{Supporting information}

Additional Supporting Information may be found in the online version of this article at the publisher's web-site:

Fig. S1. Distribution of resistance traits for seven antibiotics with significant spatial structure, as assessed by likelihood and Pearson's chi-square tests across seven collection sites (Sonoma Coast State Park isolates included in Bodega Marine Reserve collection site). Seven collection sites are designated with red dots while blue dots mark well-known California cities for geographical orientation. Pie charts at collection sites indicate the proportion of isolates from each site that exhibit resistance at a given concentration. (A) ciprofloxacin (B) kanamycin (C) neomycin (D) spectinomycin $(E)$ tetracycline $(F)$ carbenicillin $(G)$ penicillin.

Fig. S2. Relative transition rates as calculated by BayesTraits. Four states are possible for two binary characters. Arrows indicate transition direction and $q$ values indicate relative frequency of each transition. (A) Transition rates nodulation capacity and hygromycin $B$ resistance; (B) transition rates for nodulation capacity and neomycin resistance; (C) transition rates for abundance and neomycin resistance. Table S1. Haplotype designations and GenBank accession numbers for 417 isolates genotyped for glnII, ITS and recA loci. Abundance is the absolute number of times the combined gInII, ITS and recA haplotype was collected out of the total 417 isolates (a) 98 isolates chosen for antibiotic resistance assays, (b) isolates dropped after testing independence of isolates. Each isolate was deposited individually as sequencing of additional loci may reveal that isolates with identical concatenated haplotypes for glnII, ITS and recA are not clonal.

Table S2. Information on all 98 isolates. Isolates number 83-98 were excluded from further analyses as described in the 'Independence of isolates within identical haplotypes' results. Antibiotic resistance level: $0=$ sensitivity at all concentrations, while $5=$ resistance at all four tested concentrations. (a) Sonoma Coast State Park isolates were grouped with Bodega Marine Reserve isolates for all analyses; (b) nodulation capacity was assessed by PCR amplification of symbiosis island loci nifD and nodD-A all other isolates assessed with nodulation assays; (c) Abundance is the absolute number of times the combined gInll, ITS and recA haplotype was collected out of the total 417 isolates.

Table S3. Standard diversity indices.

Table S4. FST Distance method: Pairwise difference; $0=$ panmixis; 1 = complete differentiation the range 0.0 to 0.05 may be considered as indicating little genetic differentiation 0.05 to 0.15 indicates moderate genetic differentiation 0.15 to 0.25 indicates great genetic differentiation $>0.25$ indicate very great genetic differentiation.

Table S5. FST $P$-values. Number of permutations $=110$.

Table S6. Likelihood and Pearson's chi-squared tests for haplotype and antibiotic independence. Variation was calculated as the sum of individual absolute differences in the five 
level antibiotic resistance scores. All possible combinations, without replacement, were chosen using a random number generator (Haahr, 1998). Data were analysed using the 'Tests' function of 'fit $y$ by $x$ ' in JMP (SAS Institute Inc, 1989), analogous to an ANOVA (but using ordinal data). Chi-squared tests utilized sums of the absolute difference in the five-level antibiotic resistance scores for pairwise comparisons. *Significant at the $P<0.05$ level; ${ }^{* *}$ significant at the $P<0.01$ level.

Table S7. Phylogenetic signal was assessed using both Pagel's lambda and Fritz Purvis' D, first on a chromosome gene tree and subsequently on a symbiosis island gene tree if phylogenetic signal was not evident in the chromosome. Pagel's $\lambda$ ranges from zero to one: a value of 0 implies trait evolution independent of the phylogeny and $\lambda$ value of 1 indicates trait evolution is proportional to relatedness. Fritz Purvis' $D$ is one if the distribution of a binary trait is random and 0 under a Brownian motion model of evolution. Pagel's $\lambda$ was estimated using the 'fitDiscrete' function in the Geiger package in R (Harmon et al., 2007) and Fritz and Purvis' D was estimated using the 'phylo.d' function in the R package 'caper' (Orme, 2012). Phylogenetic signal was statistically significant for only the reconstructed phylogenetic tree of chromosome loci; *significant phylogenetic signal at the
$P<0.05$ level, ${ }^{* *}$ significant phylogenetic signal at the $P<0.01$ level.

Table S8. Results of likelihood and Pearson's chi-squared tests for significant spatial structure in resistance traits. *Significant at the $P<0.05$ level; ${ }^{* *}$ significant at the $P<0.01$ level.

Table S9. Results of tests for correlated evolution between nodulation capacity and antibiotic resistance traits. *Significant at the $P<0.05$ level; ** significant at the $P<0.01$ level.

Table S10. Results of tests for correlated evolution between abundance and antibiotic resistance traits. *Significant at the $P<0.05$ level; ${ }^{* *}$ significant at the $P<0.01$ level.

Table S11. Results of tests for correlated evolution between colony size and antibiotic resistance. *Significant at the $P<0.05$ level; **significant at the $P<0.01$ level. Isolates were cultured on six replica plates ( $50-150 \mathrm{CFU} /$ plate), were incubated at $29^{\circ} \mathrm{C}$, and colony size was recorded at $360 \mathrm{~h}$ (MAG, YM) or $480 \mathrm{~h}$ (GRDM) of incubation using photographs on gridded paper in ImageJ (Schneider et al., 2012). Plates were analysed if there were at least 10 distinct colonies, at least $2 \mathrm{~mm}$ from the edge of the plate or other colonies.

Appendix S1. Detailed methods for isolation protocol and phylogenetic tree reconstruction. 